> La vitamine $D$, considérée comme une véritable hormone, est essentielle au maintien de I'homéostasie phosphocalcique de l'organisme. Sa biosynthèse, tout comme sa dégradation, sont assurées par des enzymes de type cytochromes P450. La régulation de ces enzymes fait intervenir des hormones qui répondent à des variations de l'homéostasie calcique et des facteurs, appelés récepteurs nucléaires, qui modulent leur expression génique. Les affections liées à une hypovitaminose ou à une déficience métabolique (rachitisme, ostéomalacie, ostéoporose) illustrent le rôle crucial de la vitamine D dans la minéralisation osseuse et l'absorption du calcium. La découverte récente de son rôle physiologique dans la neuroprotection, l'immunité, la différenciation et la prolifération cellulaires justifie un intérêt grandissant pour cette hormone. Ainsi, une meilleure compréhension des différents acteurs impliqués dans son métabolisme et sa régulation constitue aujourd'hui un enjeu majeur pour mieux apprécier le rôle de cette vitamine. <

\section{Vitamine D : \\ métabolisme, \\ régulation \\ et maladies \\ associées}

Emilie Tissandié, Yann Guéguen,

Jean-Marc A. Lobaccaro,

Jocelyne Aigueperse, Maâmar Souidi

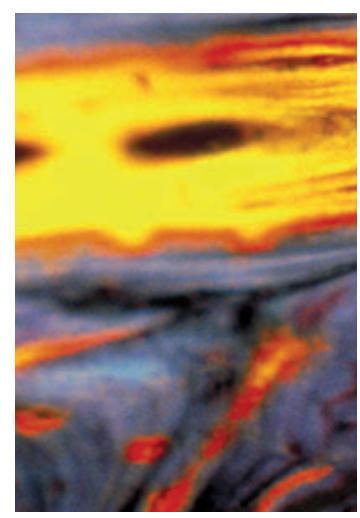

દ. Tissandié, Y. Guéguen,

J. Aigueperse, M. Souidi :

Institut de radioprotection

et de sûreté nucléaire (IRSN),

Département

de radioprotection de l'Homme,

Service de radiobiologie

et d'épidémiologie,

Laboratoire de radiotoxicologie

et in vivo ont démontré l'existence de ces rétrocontrôles et ont permis une meilleure compréhension des affections osseuses et endocriniennes liées au statut vitaminique $D$ comme le rachitisme et l'ostéomalacie. Ces expérimentale, BP 17, 92262 Fontenay-aux-Roses Cedex, France.

J.M.A. Lobaccaro: Physiologie comparée et endocrinologie moléculaire, UMR CNRS-

Université Blaise Pascal 6547, 24, avenue des Landais, 63177 Aubière Cedex, France. maamar.souidi@irsn.fr de l'homéostasie phosphocalcique de l'organisme. C'est la forme active de cette vitamine, la 1,25-dihydroxyvitamine D, qui augmente la capacité d'absorption du calcium (et du phosphore) par l'intestin, diminue sa fuite urinaire et mobilise le calcium osseux. Cette hormone présente également une activité de régulation de la défense immunitaire ainsi que la capacité de moduler la différenciation et la prolifération de certains types cellulaires. La biosynthèse et la dégradation de la vitamine $D$ sont assurées par des enzymes de type cytochromes P450 (CYP). La régulation de son métabolisme a été très étudiée au cours de ces dernières années. Les variations de la calcémie entraînent la libération d'hormones agissant sur ces CYP. La vitamine D agit aussi directement sur sa propre synthèse par une boucle de régulation négative via son récepteur nucléaire VDR (vitamin $D$ receptor). De nombreuses études in vitro

maladies résultent

principalement du défaut d'exposition solaire et des carences d'apport alimentaire en vitamine D. Elles peuvent également être induites par l'action de médicaments sur le récepteur nucléaire PXR (pregnane $X$ receptor) et, plus rarement, par des anomalies géniques du récepteur de la vitamine $D$ ou d'une des enzymes de la biosynthèse de l'hormone.

\section{Métabolisme de la vitamine D}

Le terme de «vitamine $D$ » recouvre deux composés. L'ergocalciférol, ou vitamine $D_{2}$, est présent dans l'alimentation d'origine végétale (céréales mais également

Article reçu le 12 décembre 2005, accepté le 5 mai 2006. 
champignons, levures). Le cholécalciférol, ou vitamine $D_{3}$, est produit par la peau sous l'action des rayons ultraviolets mais on le trouve également dans des aliments d'origine animale (poissons gras, aliments lactés

enrichis) [1]. Les vitamines $D_{2}$ et $D_{3}$ sont utilisées dans la prévention et le traitement curatif du rachitisme. Compte tenu de l'importance de la synthèse endogène et des faibles concentrations d'ergocalciférol dans l'alimentation, les principaux dérivés proviennent de la vitamine $D_{3}$ d'origine endogène.

\section{Biosynthèse de la vitamine $D_{3}$}

Cette biosynthèse (Figure 1) est initiée principalement dans la peau où les rayons UVB réagissent avec le 7 -déhydrocholestérol (provitamine $D$ cutanée) pour produire la pré-vitamine $D_{3}$, qui est isomérisée en cholécalciférol (ou vitamine $D_{3}$ ). Son activation est catalysée par des Cyp localisées dans les cellules hépatiques et rénales [1]. La première étape est une hydroxylation en position 25 qui conduit à la formation de 25-hydroxyvitamine $D_{3}\left(25(\mathrm{OH}) D_{3}\right)$, forme de réserve de la vitamine $D_{3}$, et dont la demi-vie plasmatique est de deux à trois semaines. Cette hydroxylation hépatique est réalisée par des CyP situées dans le réticulum endoplasmique ou dans les mitochondries. Aujourd'hui, la CYP2R1 localisée dans les microsomes apparaît comme le candidat majeur à la synthèse de $25(\mathrm{OH}) \mathrm{D}_{3}$. En effet, les individus porteurs d'une mutation du gène de la CYP2Rl possèdent

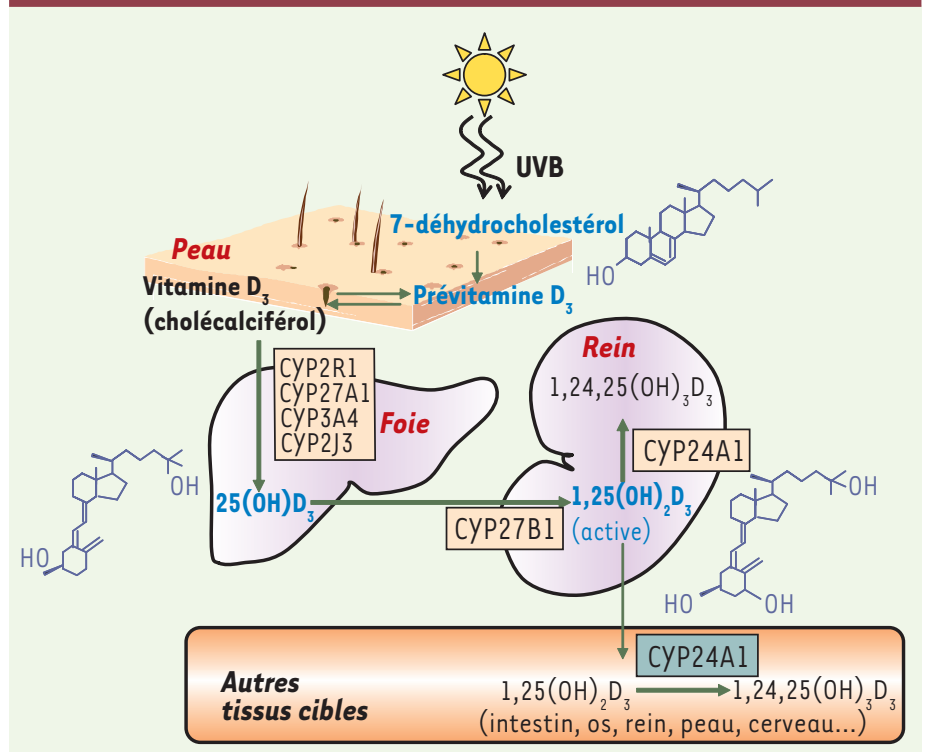

Figure 1. Schéma du métabolisme de la vitamine D3. Dans la peau, le précurseur de la vitamine $D_{3}$, le 7-déhydrocholestérol, est transformé en pré-vitamine $D_{3}$ qui est secondairement isomérisée en vitamine $D_{3}$ (ou cholécalciférol). Dans le foie, la 25-hydroxyvitamine $D_{3}$ ou $25(\mathrm{OH}) D_{3}$ est synthétisée à partir de la vitamine $D_{3}$ après action de CYP27A1, CYP2R1, CYP2J3 ou CYP3A4. Dans les tissus cibles, la $1 \alpha$-hydroxylase CYP27Bl synthétise la forme biologiquement active 1,25-dihydroxyvitamine $\mathrm{D}_{3}$ ou $1,25(\mathrm{OH})_{2} \mathrm{D}_{3}$. Son catabolisme (essentiellement dans le rein) est initié par la 24-hydroxylase CyP24Al. un taux circulant de $25(\mathrm{OH}) \mathrm{D}_{3}$ anormalement bas [2]. Cependant, la CYP27Al mitochondriale, qui intervient dans la biosynthèse des acides biliaires [3], la CYP2J3 et la CYP3A4 microsomales peuvent également catalyser cette hydroxylation [4]. Du fait de l'identification encore trop récente de la CYP2R1, peu de données sont disponibles sur cette enzyme dans la littérature. Ainsi, la suite de cette revue décrira essentiellement la CyP27Al qui est la première enzyme identifiée pouvant réaliser cette première étape hépatique. La $25(\mathrm{OH}) \mathrm{D}_{3}$ est ensuite prise en charge par la protéine plasmatique DBP (vitamin D binding protein) afin d'être véhiculée jusqu'au rein. L'endocytose du complexe $25(\mathrm{OH}) \mathrm{D}_{3} /$ DBP via la mégaline est l'une des voies d'entrée dans la cellule rénale du tube contourné proximal [5]. Les animaux ayant un défaut du gène codant la mégaline maintiennent spontanément un niveau de $1,25(\mathrm{OH})_{2} \mathrm{D}_{3}$ suffisant pour ne pas développer de rachitisme ou d'hyperparathyroïdie secondaire en cas d'apport normal de vitamine $D$, suggérant une entrée normale de $25(\mathrm{OH}) \mathrm{D}_{3}[6]$. La seconde étape est une hydroxylation en position 1 par la CYP27Bl mitochondriale qui conduit à la 1,25-dihydroxyvitamine $\mathrm{D}_{3}\left(1,25(\mathrm{OH})_{2} \mathrm{D}_{3}\right)$, forme biologiquement active, dont la demi-vie plasmatique est d'environ quatre heures.

À côté de cette production rénale majeure, des sites mineurs de production de $1,25(\mathrm{OH})_{2} \mathrm{D}_{3}$ ont été identifiés dans le placenta, le cerveau, la prostate, les kératinocytes, les ostéoblastes et les macrophages qui expriment CYP27B1. Cependant, cette production extrarénale ne contribue pas habituellement à la formation de $1,25(\mathrm{OH})_{2} \mathrm{D}_{3}$ plasmatique [7].

Une fois synthétisée, la vitamine $D_{3}$ active diffuse dans l'organisme et agit sur ses organes cibles tels que l'intestin, l'os, les reins et les parathyroïdes. D'autres sites d'action ont été identifiés : l'épiderme où elle participe au maintien de l'intégrité du tissu en agissant sur la synthèse d'involucrine, une protéine majeure de la membrane cornée des kératinocytes; le système nerveux central où elle assure une action protectrice par la synthèse de facteurs neurotrophiques. L'existence d'un métabolisme de la vitamine $D_{3}$ propre à ces deux organes fait suspecter une action autocrine et/ou paracrine dans ces tissus [8-10].

\section{Catabolisme de la vitamine $D_{3}$}

La concentration circulante en $1,25(\mathrm{OH})_{2} \mathrm{D}_{3}$ (vitamine $\mathrm{D}$ active) dépend également de son catabolisme (Figure 1) réalisé dans des cellules cibles. La CYP24Al catalyse la conversion de $1,25(\mathrm{OH})_{2} \mathrm{D}_{3}$ en 1,24,25-trihydroxyvitamine $D_{3}\left(1,24,25(\mathrm{OH})_{3} D_{3}\right)$, première étape dans la voie de dégradation de la vitamine $D$ pour aboutir à une 
forme inactive, l'acide calcitroïque. Contrairement à CyP27Al et CyP27B1, localisées principalement dans le foie et le rein respectivement, CyP24Al est ubiquitaire, contrôlant ainsi le taux de vitamine $D_{3}$ active à l'échelle de l'organisme.

\section{Régulation du métabolisme de la vitamine D}

La régulation du métabolisme de la vitamine $D_{3}$ dépend essentiellement des enzymes impliquées dans sa synthèse (CyP27Al et $\mathrm{Bl}$ ) ou son catabolisme (CyP24Al). Cette régulation fait intervenir des hormones (surtout la PTH ou parathormone) qui répondent à des variations de l'homéostasie calcique et des molécules d'origine lipidique ayant une activité autocrine ou paracrine via des récepteurs nucléaires (Figure 2).

\section{Régulation de la synthèse}

La concentration circulante de $25(\mathrm{OH}) \mathrm{D}_{3}$ est peu régulée. En effet, plus la quantité de vitamine $D$ synthétisée ou ingérée est grande, plus la production de $25(\mathrm{OH}) \mathrm{D}_{3}$ est importante. Néanmoins, dans le foie, CyP27Al, impliquée dans sa synthèse, est modulée à l'étape transcriptionnelle par des récepteurs nucléaires [1114]. On peut citer, entre autres, PPAR $\alpha$ et $\gamma$, dont les ligands sont des acides gras poly-insaturés, HNF4 $\alpha$ activé par des phosphorylations et SHP, un récepteur nucléaire ayant une activité de répression transcriptionnelle.

Dans le rein, l'activité de la CYP27B1, responsable de la production de l'hormone active $\left(1,25(\mathrm{OH}) \mathrm{D}_{3}\right)$, est étroitement régulée. La PTH libérée par les glandes parathyroïdes lors d'une hypocalcémie exerce un contrôle positif. À l'inverse, une hypercalcémie, une hypophosphatémie et/ou une augmentation de la concentration plasmatique en $1,25(\mathrm{OH})_{2} \mathrm{D}_{3}$ inhibent la libération de PTH. De plus, les phosphates, le calcium et la $1,25(\mathrm{OH})_{2} \mathrm{D}_{3}$ peuvent également agir directement sur l'enzyme et donc sur le taux circulant de I'hormone active $[1,3]$. Cependant, les mécanismes moléculaires précis sont mal connus à ce jour. Le mécanisme d'autorégulation par la $1,25(\mathrm{OH})_{2} \mathrm{D}_{3}$ sera développé ci-dessous. La PTH intervient en augmentant l'activité du promoteur de la CyP27Bl via la phosphorylation du facteur de transcription CREB ( $C A M P$-dependent response element binding protein) [15]. De nombreux autres facteurs comme I'IGF-I (insulin-like growth factor 1), I'insuline, Ia calcitonine (hormone produite par les cellules $C$ de la thyroïde), le FGF 23 (fibroblastic growth factor 23) interviennent également dans la régulation de la cyp27B1 $[1,8,16,17]$.

\section{Régulation du catabolisme}

La dégradation de la vitamine $D_{3}$ dépend, dans les reins, de la régulation de la CYP24Al. Les apports en phosphates et la PTH modulent l'activité et l'expression de cette enzyme de manière opposée à leur effet sur CyP27B1 (Figure 2). De plus, une étude récente propose une régulation par la calcitonine de l'expression du gène codant CYP24Al via la voie de signalisation Ras-PKC zêta (protéine kinase C d'isoforme zêta) [18] (Figure 2). Néanmoins, le principal facteur de transcription impliqué dans la régulation du gène codant la 24-hydroxylase est le récepteur «classique » de la vitamine $D_{3}$, VDR (vitamin D receptor). Cette protéine appartient à la superfamille des récepteurs nucléaires, facteurs de transcription activés par des ligands (hormones ou molécules lipophiles). La $1,25(\mathrm{OH})_{2} \mathrm{D}_{3}$ stimule la transcription de CyP24Al via sa fixation sur l'hétérodimère formé par VDR et RXR (retinoic $X$ receptor), le récepteur de l'acide rétinoïque 9-cis, qui reconnaît des séquences spécifiques (séquences VDRE, vitamin $D$ response element) dans le promoteur du gène [19] (Figure 3). Par ailleurs, VDR aurait un rôle direct dans l'inhibition de l'expression de la CYP27Bl dans les reins. Cette répression résulterait de la liaison de l'hétérodimère VDR/RXR à un facteur de trans-

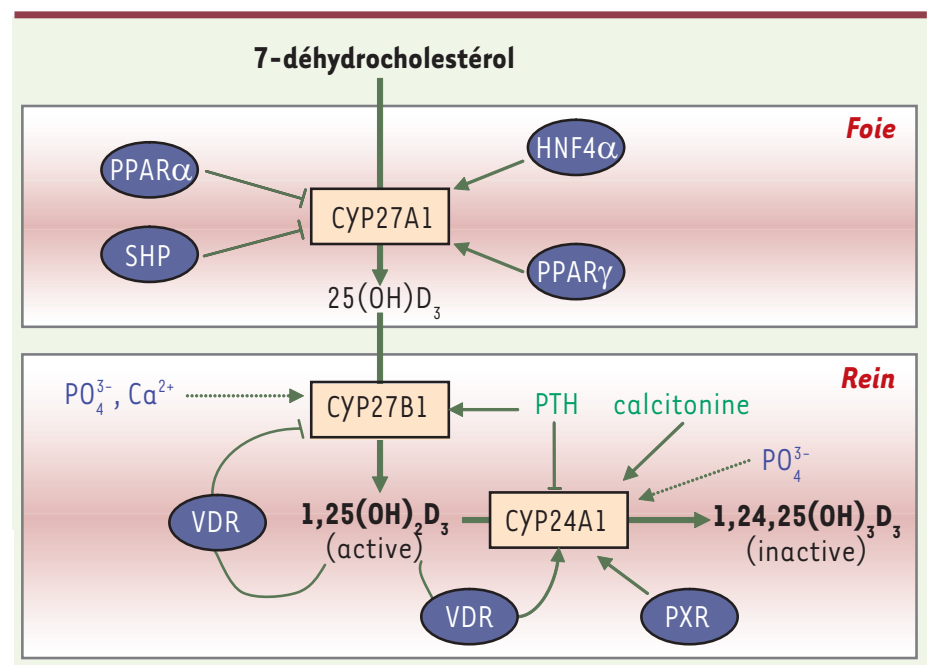

Figure 2. Régulation du métabolisme de la vitamine D3 par les hormones, les minéraux et les récepteurs nucléaires. Dans le foie, l'expression de la CYP27Al est stimulée par les récepteurs nucléaires HNF4 $\alpha$ (hepatic nuclear factor $4 \alpha$ ) et PPAR $\gamma$ (peroxisome proliferator-activated receptor $\gamma$ ) et inhibée par PPAR $\alpha$ et SHP (small heterodimer partner). Dans le rein, la parathormone (PTH) est le régulateur positif principal de la CYP27B1, enzyme responsable de la production de la vitamine $D_{3}$ active. L'hypocalcémie et l'hypophosphatémie induisent une augmentation de l'activité et de l'expression de cette enzyme, alors que l'hypercalcémie et l'hyperphosphatémie exercent un contrôle négatif. La $1,25(\mathrm{OH})_{2} \mathrm{D}_{3}$, elle même, via son interaction avec VDR (vitamin D receptor) inhibe l'expression rénale de la CYP27Bl et stimule la transcription de la CYP24Al rénale, responsable de l'inactivation de la vitamine $D_{3}$. La CYP24Al est régulée également par les apports phosphatés et par la PTH. Cette dernière inhibe l'expression de l'enzyme. Au contraire, la calcitonine et le récepteur nucléaire PXR (pregnane $\chi$ receptor) induisent son expression. Flèche droite, stimulation; flèche brisée, inhibition; flèche en pointillée : faible action de régulation. 
cription de type bHLH (basic helix-loop-helix) capable d'interagir avec un motif consensus appelé «boîte $\varepsilon$ » présent sur le promoteur de la CYP27B1 [20]. Via l'action de VDR, la $1,25(\mathrm{OH})_{2} \mathrm{D}_{3}$ peut exercer un contrôle sur sa propre synthèse et son propre catabolisme. Un autre récepteur nucléaire, PXR (pregnane $X$ receptor), impliqué dans la régulation du métabolisme des xénobiotiques et des médicaments, permettrait l'induction du gène codant CYP24Al grâce à son interaction avec les séquences VDRE [21] (Figure 2). Ainsi, les médicaments anti-épileptiques ou anti-convulsifs, activateurs de PXR, de même que les corticostéroïdes, peuvent conduire à une carence en vitamine $D$. Lorsque ce type de médicament est prescrit à long terme, comme c'est le cas pour les personnes épileptiques ou souffrant d'arthrite, un apport supplémentaire en vitamine $D$ peut s'avérer nécessaire.

\section{Rôles biologiques de la vitamine D}

La $1,25(\mathrm{OH})_{2} D_{3}$ est une hormone hypercalcémiante. Elle agit essentiellement à trois niveaux. (1) Intestinal : elle permet une absorption intestinale accrue du calcium alimentaire et secondairement celle des phosphates. Deux sites d'action sont reconnus. Sur la bordure en brosse des cellules intestinales, cette hormone augmente la synthèse du transporteur de calcium ( $\mathrm{CaTl}$ ) qui est le mode d'action majeur pour l'absorption intestinale du calcium. Dans les cellules intestinales, elle augmente la synthèse de la protéine calbindine qui favorise le transport du calcium contre un gradient de concentration entre les cellules intestinales et le plasma, entraînant ainsi la diffusion passive des ions phosphates. (2) Osseux: en réponse à une hypocalcémie, la vitamine $D$ active de façon directe la résorption osseuse en favorisant la différenciation et l'activation des cellules souches mésenchyma-

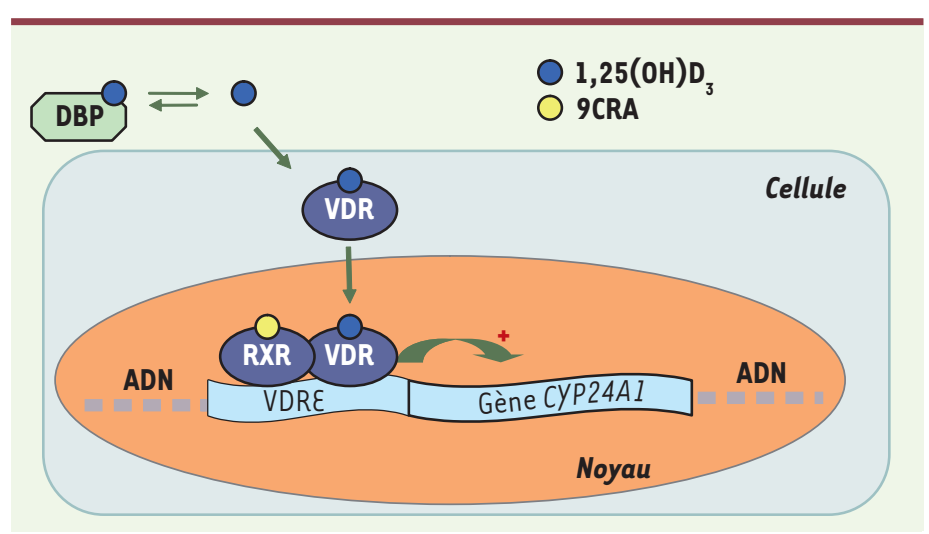

Figure 3. Schéma de la régulation transcriptionelle de l'expression de CYP24A1 par le récepteur de la vitamine $D$. La vitamine $D$ active, $1,25(\mathrm{OH})_{2} \mathrm{D}_{3}$, circule liée à $\mathrm{DBP}$ (vitamin $\mathrm{D}$ binding protein). $1,25(\mathrm{OH})_{2} \mathrm{D}_{3}$ pénètre dans la cellule cible et va se lier à son récepteur nucléaire VDR (vitamin $D$ receptor). Le complexe $1,25(\mathrm{OH})_{2} \mathrm{D}_{3}$ VDR forme un hétérodimère avec RXR (retinoic $X$ receptor) dont le ligand est l'acide rétinoïque 9-cis (9CRA). L'heterodimère VDR/RXR se lie à des séquences spécifiques (séquences VDRE, vitamin $D$ response element) situées en amont du gène de la CYP24Al. II s'ensuit une activation (+) de la transcription du gène de CYP24Al. teuses de l'os en ostéoclastes. (3) Rénal: I'hormone augmente la réabsorption tubulaire du calcium par action directe sur le canal épithélial calcique ( $\varepsilon C a C)$. Son effet stimulant sur la réabsorption tubulaire des phosphates est secondaire à l'inhibition de la sécrétion de PTH produite par l'hypercalcémie associée à l'administration de vitamine $D$. Elle accélère également le transport du calcium et des phosphates par un mécanisme dépendant de la PTH $[1,22]$.

L'action de la $1,25(\mathrm{OH})_{2} \mathrm{D}_{3}$ s'exerce via deux voies différentes. La plus connue implique la liaison de $1,25(\mathrm{OH})_{2} \mathrm{D}_{3}$ à VDR qui induit une activation ou une répression de la transcription de gènes cibles, comme décrit ci-dessus. Des études plus récentes suggèrent l'existence d'une voie dite «non génomique », plus rapide, faisant intervenir un VDR membranaire capable d'activer la voie de transduction impliquant la protéine kinase $C$ et capable de modifier le métabolisme des phospho-inositides et la distribution intracellulaire du calcium [23].

À côté de son rôle bien établi dans la régulation de I'homéostasie phosphocalcique, la vitamine $D$ possède d'autres fonctions physiologiques (Figure 4) telles que des effets immunomodulateurs ainsi qu'une implication dans le contrôle de la différenciation de nombreux types cellulaires et l'inhibition de leur prolifération [24, 25]. La mise en évidence de ces nouvelles propriétés a initié de nombreuses études concernant l'utilisation de cette hormone et de ses analogues moins hypercalcémiants dans le traitement des maladies hyperprolifératives (cancers) et dans celui des maladies auto-immunes (diabète de type 1) [26, 27]. Parmi les analogues de la vitamine $D$, on peut citer, par exemple, le calcipotriène utilisé dans le traitement du psoriasis. Enfin, il faut également noter que la $24,25(\mathrm{OH})_{2} \mathrm{D}_{3}$, un métabolite, aurait un rôle dans la croissance, le développement et la réparation des os [28].

\section{Maladies associées au métabolisme de la vitamine D}

Les maladies liées au statut vitaminique $D$ sont très souvent le résultat d'une carence en vitamine $D$. Cette carence entraîne un rachitisme chez le jeune en croissance [29]. Chez l'adulte, un trouble de la minéralisation osseuse, appelé ostéomalacie [30], peut entraîner une hypersécrétion de PTH. Chez la personne âgée, cette carence constitue un terrain favorable à l'ostéoporose, maladie caractérisée par une masse minérale basse et des altérations de la microarchitecture osseuse [31]. Les fractures sont la complication la plus fréquente et s'accompagnent d'une augmentation de la morbidité et de la mortalité. II est 
intéressant de noter que l'ostéomalacie peut résulter de la prise prolongée de médicaments tels que le phénobarbital [32], qui après activation du récepteur nucléaire PXR, induit l'expression de CYP24Al, ce qui conduit à la dégradation incontrôlée de la vitamine $D$ active entraînant une altération de l'homéostasie phosphocalcique [33].

Il existe également des maladies héréditaires du métabolisme de la vitamine $D$ à transmission autosomique récessive. Le rachitisme pseudo-carentiel de type I résulte d'une anomalie de l'expression du gène codant CyP27B1 [34]. Le taux de $25(\mathrm{OH}) \mathrm{D}_{3}$ est normal chez les individus atteints du rachitisme pseudo-carentiel de type I alors que le taux de $1,25(\mathrm{OH})_{2} \mathrm{D}_{3}$ s'effondre. Des déformations et douleurs osseuses, un retard de croissance et une myasthénie caractérisent la maladie. L'administration de $1,25(\mathrm{OH})_{2} \mathrm{D}_{3}$ est nécessaire pendant toute la vie des personnes atteintes de cette affection. A l'inverse, le rachitisme pseudo-carentiel de type II résulte d'une anomalie de l'expression du gène codant VDR. II se caractérise donc par une résistance des organes cibles à l'action de $1,25(\mathrm{OH})_{2} \mathrm{D}_{3}$ dont le taux est élevé. Ce rachitisme pseudo-carentiel de type II se manifeste par un tableau de rachitisme sévère et précoce, associé à une alopécie dans $80 \%$ des cas. Le traitement consiste en l'administration de doses massives de tous les dérivés de la vitamine $D_{3}$ et de calcium [35].

À côté de ces états de carence, de déficit ou d'insuffisance en vitamine $D$, un apport excessif (intoxication) peut entraîner une hypervitaminose qui provoque une hypercalcémie par augmentation de l'absorption intestinale et de la résorption osseuse. Cette hypercalcémie peut conduire à des atteintes rénales (lithiases et néphrocalcinoses). Les cas d'hypervitaminose endogène sont rares sauf dans certaines maladies telles que les granulomatoses qui se caractérisent par une production non contrôlée de $1,25(\mathrm{OH})_{2} \mathrm{D}_{3}[36,37]$.

\section{Conclusions}

La vitamine $D$ est connue depuis longtemps pour son rôle primordial dans le contrôle de l'homéostasie phosphocalcique et dans la minéralisation osseuse. La mise en évidence plus récente de son rôle physiologique dans l'immunité, la différenciation cellulaire, la prolifération et la neuroprotection justifie un intérêt grandissant pour cette hormone.

Au cours de ces dernières années, l'étude du métabolisme de la vitamine $D$, de son mécanisme d'action et de ses «nouveaux » tissus cibles a été abordée, notamment, par l'utilisation d'outils de biologie cellulaire et moléculaire. Les données obtenues ont mis en évidence les principaux acteurs de ce métabolisme: enzymes de type cytochromes P450 (CYP27Al, CYP2R1, CYP27Bl et CYP24Al) et récepteur nucléaire VDR qui jouent un rôle majeur dans le contrôle du métabolisme de la vitamine $D$. De façon plus surprenante, la production de vitamine $D$ a pu être mise en évidence dans d'autres tissus (placenta, cerveau, prostate, peau). Si l'existence de cette synthèse locale suggère une action autocrine et/ou paracrine de cette hormone, les cibles moléculaires et surtout les fonctions physiologiques d'une telle synthèse sont encore en cours d'étude.

Un manque de vitamine $D$ entraîne un rachitisme chez l'enfant ainsi qu'une exacerbation de l'ostéoporose et le développement d'une ostéomalacie chez l'adulte. L'allongement de la durée de vie et l'incidence des fractures provoquées par l'ostéoporose ont fait de cette affection un problème de santé publique très actuel. De plus, une grande partie de la recherche sur la vitamine $D$ a trait au développement de I'utilisation du $1,25(\mathrm{OH})_{2} \mathrm{D}_{3}$ comme agent prévenant ou retardant la survenue de certaines maladies auto-immunes (diabète de type 1) ou prolifératives (cancers solides, leucémie, psoriasis). Une meilleure compréhension du rôle des CyP impliquées dans le métabolisme de la vitamine D ainsi que de celui des facteurs régulant leur expression comme les récepteurs nucléaires et les co-facteurs associés, constitue un des défis à résoudre afin de mieux apprécier le rôle de la vitamine $D$ dans les conditions physiologiques normales ou dans les maladies associées. $\diamond$

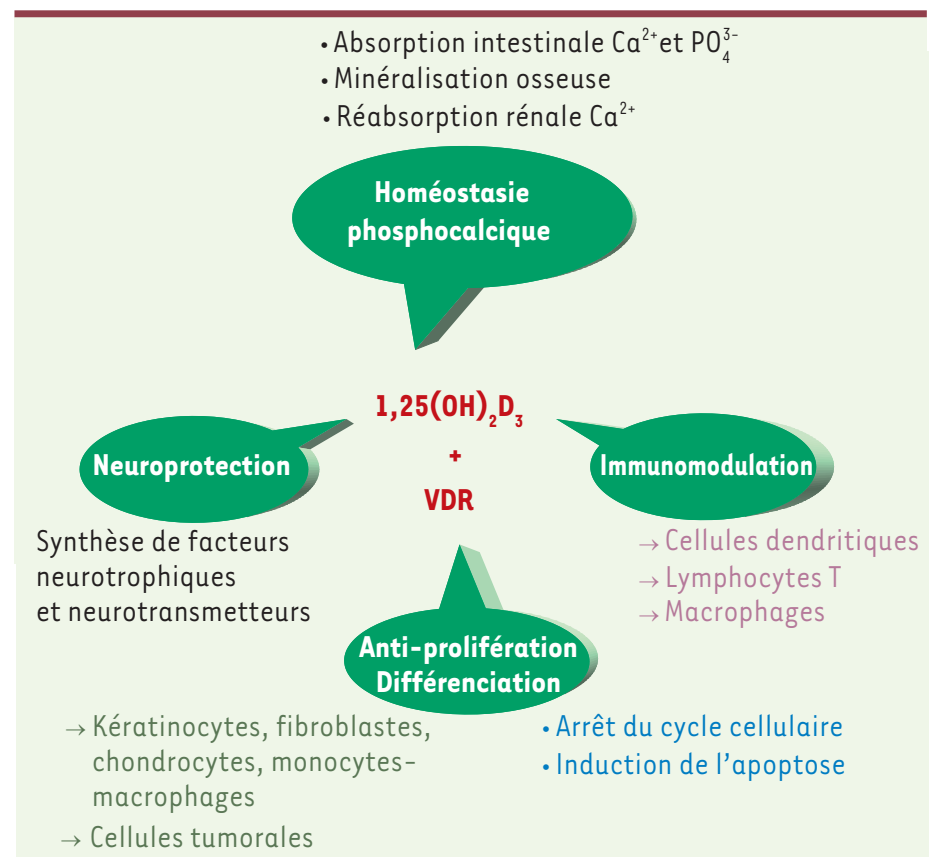

Figure 4. Rôles physiologiques de la vitamine $D_{3^{*}}$ La forme active de la vitamine $D_{3}, 1,25(\mathrm{OH}) 2 \mathrm{D} 3\left(1,25\right.$-dihydroxyvitamine $\left.D_{3}\right)$, liée à son récepteur nucléaire VDR est impliquée dans divers processus physiologiques au sein de l'organisme (pour plus de détails, se référer au texte). 


\section{SUMMARY}

Vitamin D: metabolism, regulation and associated diseases

Vitamin $D$ is well known as a hormone involved in mineral metabolism and bone growth. Conversion into the active metabolite 1,25-dihydroxyvitamin $D_{3}\left(1,25(\mathrm{OH})_{2} D_{3}\right)$ from the precursor is effected by cytochrome P450 enzymes in the liver (CYP27Al and CYP2R1) and the kidney (CYP27B1). CYP27Al has been shown to be transcriptionally regulated by nuclear receptors (PPAR $\alpha, \gamma, \mathrm{HNF}-4 \alpha$ and SHP) which are liganddependent transcription factors. CYP27Bl is tightly regulated by the plasma levels of calcium, phosphate, parathyroid hormone (PTH) and $1,25(\mathrm{OH})_{2} \mathrm{D}_{3}$ itself. In vitamin $\mathrm{D}$ target organs, inactivation of vita$\min \mathrm{D}$ is attributed to CyP24Al which is transcriptionally induced by $1,25(\mathrm{OH})_{2} \mathrm{D}_{3}$ whose action is mediated by binding to its cognate nuclear receptor, the vitamin $D$ receptor (VDR). Diseases associated to Vitamin $D$ deficiency (rickets in children, and osteomalacia or osteoporosis in adults) and autosomal recessive forms of inherited rickets illustrate the key role of vitamin $D$ in calcium homeostasis and bone metabolism. Recently, discovery of $1,25(\mathrm{OH})_{2} \mathrm{D}_{3}$ new biological actions that include antiproliferative, prodifferentiating effect on many cell types and immunoregulatory properties creates a growing interest for this vitamin. In this way, a best understanding of various actors implicated in vitamin D metabolism and its regulation is of a major importance to optimise the use of vitamin $D$ in disease prevention. $\diamond$

\section{REFERENCES}

1. Brown AJ, Dusso AS, Slatopolsky E. Vitamin D. Am J Physiol $1999 ; 277$ : F157-75.

2. Cheng JB, Levine MA, Bell NH, et al. Genetic evidence that the human CYP2R1 is a key vitamin D 25-hydroxylase. Proc Natl Acad Sci USA 2004 ; 101 : 7711-5.

3. Souidi M, Dubrac S, Parquet M, Lutton C. Hepatic and extrahepatic sterol 27-hydroxylase: roles in cholesterol and bile acid metabolism and associated diseases. Gastroenterol Clin Biol $2003 ; 27: 100-11$.

4. Prosser $D E$, Jones $G$. Enzymes involved in the activation and inactivation of vitamin $D$. Trends Biochem Sci $2004 ; 29: 664-73$.

5. Nykjaer A, Dragun D, Walther D, et al. endocytic pathway essential for renal uptake and activation of the steroid 25-(OH) vitamin D3. Cell 1999; $96: 507-15$.

6. Leheste JR, Melsen F, Wellner M, et al. Hypocalcemia and osteopathy in mice with kidneyspecific megalin gene defect. FASEB J $2003 ; 17: 247-9$.

7. Garabédian M. La 1,25dihyroxyvitamine D et son récepteur. Rev Rhum 2000 ; 67 (suppl 2) : 39-41.

8. Miller WL, Portale AA. Vitamin D 1 alpha hydroxylase. Trends Endocrinol Metab 2000 ; $11: 315-9$.

9. Garcion $\varepsilon$, Wion-Barbot N, Montero-Menei CN, et al. New clues about vitamin D functions in the nervous system. Trends Endocrinol Metab 2002; $13: 100-5$.

10. Schuessler M, Astecker N, Herzig G, et al. Skin is an autonomous organ in synthesis, two-step activation and degradation of vitamin $\mathrm{D}_{3}$ : CYP27 in epidermis completes the set of essential vitamin $D_{3}$-hydroxylases. Steroids $2001 ; 66: 399-408$.

11. Post SM, Duez H, Gervois PP, et al. Fibrates suppress bile acid synthesis via peroxisome proliferator-activated receptor-alpha-mediated downregulation of cholesterol 7alphahydroxylase and sterol 27-hydroxylase expression. Arterioscler Thromb Vasc Biol 2001 ; $21: 1840-5$.

12. Chen W, Chiang Jy. Regulation of human sterol 27-hydroxylase gene (CYP27Al) by bile acids and hepatocyte nuclear factor 4 alpha (HNF4alpha). Gene 2003 ; $313: 71-82$.

13. Quinn CM, Jessup W, Wong J, et al. Expression and regulation of sterol 27-hydroxylase (CYP27A1) in human macrophages: a role for RXR and PPARgamma ligands. Biochem / 2005 ; $385: 823-30$.
14. Eloranta JJ, Kullak-Ublick GA. Coordinate transcriptional regulation of bile acid homeostasis and drug metabolism. Arch Biochem Biophys 2005; $433: 397-412$.

15. Armbrecht HJ, Hodam TL, Boltz MA. Hormonal regulation of 25hydroxyvitamin D3-lalpha-hydroxylase and 24-hydroxylase gene transcription in opossum kidney cells. Arch Biochem Biophys 2003 ; 409: 298-304.

16. Hewison M, Zehnder D, Bland R, Stewart PM. $1 \alpha$-Hydroxylase and the action of vitamin D. Mol Endocrinol $2000 ; 25: 141-8$.

17. Schiavi SC, Kumar R. The phosphatonin pathway: new insights in phosphate homeostasis. Kidney Int $2004 ; 65$ : 1-14.

18. Gao XH, Dwivedi PP, Omdahl JL, et al. Calcitonin stimulates expression of the rat 25-hydroxyvitamin D3-24-hydroxylase (CyP24) promoter in HEK-293 cells expressing calcitonin receptor: identification of signaling pathways. J Mol Endocrinol 2004 ; 32 : 87-98.

19. Chen KS, DeLuca HF. Cloning of the human 1 alpha, 25-dihydroxyvitamin D-3 24-hydroxylase gene promoter and identification of two vitamin Dresponsive elements. Biochem Biophys Acta 1995 ; $1263: 1-9$.

20. Murayama A, Kim M, Yanagisawa J, et al. Transrepression by a liganded nuclear receptor via a bHLH activator through co-regulator switching. EMBO J $2004 ; 23$ : 1598-608.

21. Pascussi JM, Robert A, Nguyen $M$, et al. Possible involvement of pregnane $X$ receptor-enhanced CYP24 expression in drug-induced osteomalacia.J Clin Invest $2005 ; 115$ : 177-86.

22. Dusso AS, Brown AJ, Slatopolsky $\varepsilon$. Vitamin D. Am J Physiol Renal Physiol $2005 ; 289$ : F8-28.

23. Norman AW, Ishizuka S, Okamura WH. Ligands for the vitamin D endocrine system: different shapes function as agonists and antagonists for genomic and rapid response receptors or as a ligand for the plasma vitamin D binding protein. J Steroid Biochem Mol Biol $2001 ; 76: 49-59$.

24. Van $\varepsilon$ tten $\varepsilon$, Mathieu C. Immunoregulation by 1,25 -dihydroxyvitamin $D_{3}$ : Basic concepts. J Steroid Biochem Mol Biol 2005; 97 : 93-101.

25. Holick MF. Vitamin D : A millenium perspective. J Cell Biochem 2003 88: 296-307.

26. Holick MF. Noncalcemic actions of 1,25-dihydroxyvitamin D3 and clinical applications. Bone 1995; 17 (suppl 2) : S107-11.

27. Adorini L. Immunomodulatory effects of vitamin D receptor ligands in autoimmune diseases. Int Immunopharmacol 2002 ; 2 : 1017-28.

28. St-Arnaud R, Glorieux FH. 24, 25-Dihydroxyvitamin D: active metabolite or inactive catabolite? Endocrinology 1998 ; 139 : $3371-4$.

29. Garabédian M, Menn S, Walrant-Debray 0, et al. Prevention of child and adolescent vitamin $D$ deficiency. II. Validation of a decision-making abacus based on sun exposure and vitamin D intakes. Arch Pediatr 2005; $12: 410-9$.

30. Maugars Y, Glémarec J, Guillot P, et al. Métabolisme phosphocalcique et ostéomalacie. Rev Rhum $2000 ; 67$ (suppl 2) : 95-8.

31. Breuil V, Euller-Ziegler. Nutrition et vieillissement osseux : I'ostéoporose. Nutr Clin Metab 2004 ; $18: 212-8$

32. Gascon-Barré M. Antiepileptic drugs and bone health. In : DawsonHughes B, Holick MF, eds. Nutrition and bone health. Totowa-New Jersey, USA : Humana Press, $2004: 647-66$.

33. Pascussi JM, Vilarem MJ. Ostéomalacie consécutive à la prise prolongée de médicaments. Sur la piste de PXR, un récepteur impliqué dans la détoxication. Med Sci (Paris) $2005 ; 21: 582-3$.

34. Kato S, Yoshizazawa T, Kitanaka $S$, et al. Molecular genetics of vitamin D- dependent hereditary rickets. Horm Res 2002 ; 57 : 73-8.

35. Dumas R. Les rachitismes vitaminorésistants. Arch Pediatr 1999 ; $6: 715-9$.

36. Souberbielle JC, Friedlander G, Kahan A, Cormier C. Evaluating vitamin D status. Implications for preventing and managing osteoporosis and other chronic diseases. Joint Bone Spine $2006 ; 73: 249-53$.

37. Holick MF. Defects in the synthesis and metabolism of vitamin D. Exp Clin Endocrinol Diabetes $1995 ; 103: 219-27$

\section{TIRÉS À PART}

\section{દ. Tissandié}

